\title{
Age-related differences in the morphology of the impedance cardiography signal
}

\author{
Christian Tronstad ${ }^{1,3}$, Jan Olav Høgetveit ${ }^{1,2}$, Ole Elvebakk ${ }^{1}$ and Håvard Kalvøy ${ }^{1}$ \\ 1. Department of Clinical and Biomedical Engineering, Oslo University Hospital, Oslo, Norway \\ 2. Department of Physics, University of Oslo, Oslo. Norway \\ 3. E-mail any correspondence to: christian.tronstad@gmail.com
}

\begin{abstract}
Impedance cardiography (ICG) is a non-invasive method of hemodynamic measurement, mostly known for estimation of stroke volume and cardiac output based on characteristic features of the signal. Compared with electrocardiography, the knowledge on the morphology of the ICG signal is scarce, especially with respect to age-dependent changes in ICG waveforms. Based on recordings from ten younger (20-29 years) and ten older (60-79) healthy human subjects after three different levels of physical activity, the typical interbeat ICG waveforms were derived based on ensemble averages. Comparison of these waveforms between the age groups indicates the following differences: a later initial upward deflection for the younger group, an additional hump in the waveform from many older subjects not presented in the younger group, and a more pronounced second wave in the younger group. The explanation for these differences is not clear, but may be related to arterial stiffness. Further studies are suggested to determine whether these morphological differences have clinical value.
\end{abstract}

Keywords: Bioimpedance; impedance cardiography; age; signal analysis

\section{Introduction}

Hemodynamic measurements often include invasive catheter-based techniques like thermodilution [Nyboer, 1940] or advanced imaging modalities like Ultrasounddoppler [Thiele, 2015] or Magnetic Resonance Imaging (MRI) [Chai 2005]. The risks of catheter-based methods are well-documented and include bloodstream infections and sepsis and are associated with increased morbidity [Bouza, 2007; Linares, 2007]. Ultrasound Doppler-based methods are dependent on precise alignment of the beam and the aortic cross-sectional area [Thiele, 2015], while MRI-based methods are time-consuming and expensive [Li, 2015; Young, 2015].

Impedance cardiography (ICG) is a non-invasive, low cost alternative to the aforementioned methods and was described in 1940 by Nyboer et al. [1940]. Nyboer found that the beat-to-beat stroke volume (SV) is given by the segment length of the chest (L), the resistivity of blood $(\rho)$, the maximum backward extrapolated value of the thoracic cardiogenic impedance change $\left(\Delta Z_{\max }\right)$ and the base impedance $\left(Z_{0}\right)$ by the following equation:

$$
V=\rho \frac{L^{2}}{Z_{0}^{2}} \Delta Z_{\max }
$$

The model was further developed by Kubicek et al. [1966] to include the left ventricular ejection time (TLVE), the stroke volume is given by the equation:

$$
S V=\rho \frac{L^{2}}{Z_{0}^{2}}\left(\frac{d Z}{d t}\right)_{\max } T_{L V E}
$$

Sramek et al. replaced the cylindrical model of the chest with a truncated cone and an estimated a fixed chest length of $17 \%$ of the total height [Sramek, 1983; Van De Water, 2003], a model that was refined by Bernstein including a new parameter $\delta$ which is the actual weight divided by the ideal weight [Bernstein, 1986]:

$$
S V=\delta \cdot\left(\frac{L^{3}}{4,25}\right) \cdot \frac{\left(\frac{d Z}{d t}\right)_{\max }}{Z_{0}} \cdot T_{L V E}
$$


Bernstein later extended the model with a new parameter $\zeta$ (zeta), which is an index of transthoracic aberrant conduction, in order to compensate for the conduction through the very highly conductive interstitial extravascular lung water [Bernstein, 2010]. The relation between the volume of electrically participating thoracic tissue (VEPT), the intrathoracic blood volume (VITBV) and $\zeta$ is given by:

$$
V_{E P T}=\frac{V_{I T B V}}{\zeta^{2}}
$$

This ends up with the Bernstein stroke volume equation for impedance cardiography [Bernstein, 2010]:

$$
S V=\frac{V_{I T B V}}{\zeta^{2}} \sqrt{\frac{\left(\frac{d Z}{d t}\right)_{\max }}{Z_{0}}} \delta \cdot T_{L V E}
$$

ICG has been compared to a number of different methods for measuring cardiac output. These studies are not as rigorously designed as the studies for thermodilution and Doppler-based ultrasound techniques, but the majority of the studies indicate lower accuracy for ICG than the competing methods [Thiele, 2015]. The level of adequacy of hemodynamic measurement techniques is not welldefined; it depends on the procedure and is a clinical discussion. However, describing and testing applications in the outer limit of the range will provide some important information about the limitations and possibilities of the method. Lately, hemodynamic patterns identified by ICG has been shown to predict mortality in the general population [Medina-Lezama et al., 2018].
The use of ICG for cardiac output measurements is a well-known method but little is known of the morphology of the ICG signal compared to signals from other cardiovascular measures such as continuous blood pressure and ECG.

A typical human ICG curve with marked characteristic points is shown in Bernstein [2009] and is reproduced below in figure 1 , along with definitions of characteristic point of the ICG curve and their physiological attribution.

Although the ICG curve is defined in the literature, the shape of representative ICG curves of apparently healthy subjects can have substantial differences between publications, comparing figure 1 to figures in for example [Cybulski, 2011; Marquez et al., 2013; Carvalho et al. 2011]. The issue of differences in ICG waveforms and complexes of characteristic points is very well highlighted in Ermishkin et al. [2012] and in Benouar et al. [2018], also demonstrating that the ICG waveform morphology is far from stationary.

Compared with ECG, the knowledge on the morphology and interpretation of the ICG signal is still scarce, especially with respect to age-dependent changes in ICG waveforms. ICG measurement provides a non-invasive recording that is different from both ECG and optical plethysmographic methods, and could possibly provide physiological information that is not provided by other non-invasive methods, or improve predictions in combination with them. Aging is known to bring changes in the cardiovascular physiology such as arterial stiffness and hypertension [Sun, 2016], and it is likely that the ICG waveform is altered along with these age-related changes.

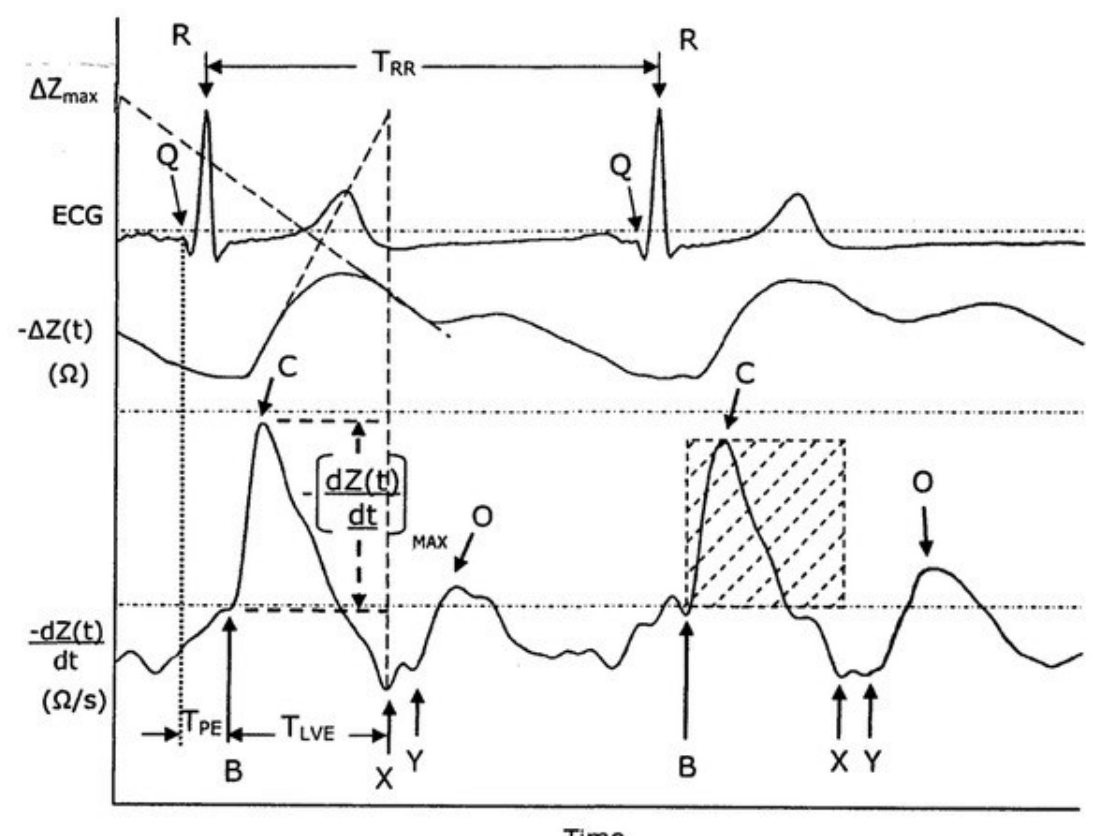

Time

Fig. 1: ICG plotted as change in impedance $(-\Delta Z)$, the time-derivative of the impedance (-dZ/dt) together with ECG. The B point marks the aortic valve opening, the $X$ point marks aortic valve closure, the $Y$ point marks pulmonic valve closure and the $O$ point marks the rapid ventricular filling wave. The distance from the ECG Q-point to the ICG B point is attributed to the pre-ejection period of the heart (TPE), and the ICG B-X distance is attributed to the left ventricular ejection time (TLVE). The figure is copied from Bernstein 2009 under the Wikimedia Commons license. 
The aim of this study was to derive the average intrabeat ICG waveform in two separate groups of younger and older subjects and inspect possible differences in ICG morphology between the groups. Further on, the aims are to interpret any differences with respect to cardiovascular physiology based on existing literature and suggestions for further studies. In the end, possible ICG patterns related to aging and cardiovascular risks can be considered for clinical use.

Materials and methods

Dataset

From an earlier study on ICG for estimation of pulse wave velocity [Aria et al., 2019], an anonymized dataset of ICG and electrocardiography (ECG) recordings from 20 healthy volunteers in two age groups (20-29 and 60-79 years) was used in this study. Both age groups had 10 participants with five males and five females. Briefly, ICG (dz/dt) over the thorax and at the upper arm was recorded simultaneously with ECG for one minute following three different levels of physical activity using a stationary bike at low (phase 1), intermediate (phase 2) and high (phase 3) mechanical resistance. More details on the protocol and the ICG measurement can be found in Aria et al. [2019]. Only the ICG recordings over the thorax were used in this study, together with the ECG recordings. The ICG and ECG recordings were sampled synchronously with a rate of 2000 samples per second.

\section{Signal processing}

In order to study the ICG waveform, each heartbeat interval within every recording was identified by the location of the $\mathrm{R}$ peaks from the corresponding ECG recording, extracting ICG beats between all successive $R$ peaks. The PanTompkins algorithm [Pan \& Tompkins, 1985] was used for automatic identification of the $R$ peaks in from the ECG recording. The ICG beats were then resampled to a length of one second (2000 samples).

\section{Waveform analysis}

For each recording, the ensemble average of all ICG beats were used to derive the typical waveform for that participant and physical activity level. The ensemble averages were then grouped into the two age groups and a grand ensemble average was calculated based on a mean of these for all three physical activity levels, enabling a comparison of the ICG morphology between the two age groups. Although the $\mathrm{dz} / \mathrm{dt}$ signal has been plotted with the largest deflection (C-point) in the downward direction in earlier publications [Van Eijnatten et al, 2014; Aria et al. 2019], the negative $\mathrm{dZ} / \mathrm{dt}$ (with an upward deflecting Cwave) has been plotted here for easier comparison with other studies. In order to investigate changes in the direction of the $-d z / d t$ curve such as small humps, the second derivative of the impedance signal $\left(-d^{2} \mathrm{Z} / \mathrm{dt}^{2}\right)$ was also calculated and presented in ensemble averages.

\section{Informed consent}

Informed consent has been obtained from all individuals included in this study.

\section{Ethical approval}

The research related to human use has been complied with all relevant national regulations, institutional policies and in accordance with the tenets of the Helsinki Declaration, and has been approved by the authors' institutional review board or equivalent committee.

\section{Results}

As shown in figure 2, the shape of the ICG waveform had substantial interindividual variation. It can also be seen from the figure that some recordings had a lower repeatability from beat to beat than the rest (e.g. upper left plot in figure 1a). In most of the cases, the waveform was similar for the same individual over the three phases.

One ICG recording from phase 3 was excluded from analysis due to errors in the ECG recording.

The grand ensemble averages (mean of the ensemble averages) of $-d z / d t$ for both age groups and each phase is shown in figure 3 , allowing the interpretation of waveform differences between younger and older participants based on visual inspection. The curves show a tendency of later initial upward deflection for the younger subjects, and for the older group, an additional hump in the following downward deflection around sample nbr 400 that is not present in the younger group. The second wave at around sample nbr 1000 is more pronounced in the younger group compared to being almost invisible in the grand ensemble average ICG of the older group. For enhancement of bumps in the waveform, the second derivative ICG $\left(-d^{2} \mathrm{Z} / \mathrm{dt}^{2}\right)$ is presented in figure 4 . This presentation shows the bump around sample nbr 400 more clearly as a small wave in the second derivative that is not present in the grand ensemble average from the younger group.

\section{Discussion}

The analysis showed a difference in the average ICG waveform between younger and older healthy individuals, indicating a possible effect of age on the morphology of the ICG. It is well known that aging affects the cardiovascular system, such as stiffness of the arteries [Sun, 2016]. It is known based on the previous study by Aria et al. [2019] using the same data, that the pulse wave velocity was higher for the older subjects, indicating increased stiffness of the arterial walls, which may have influenced the ICG waveform. The earlier rise in the ICG waveform for the older subjects could be attributed to a shorter pre-ejection period, attributed to the contractility of the heart regulated by sympathetic activity [Van Eijnatten et al., 2014]. 

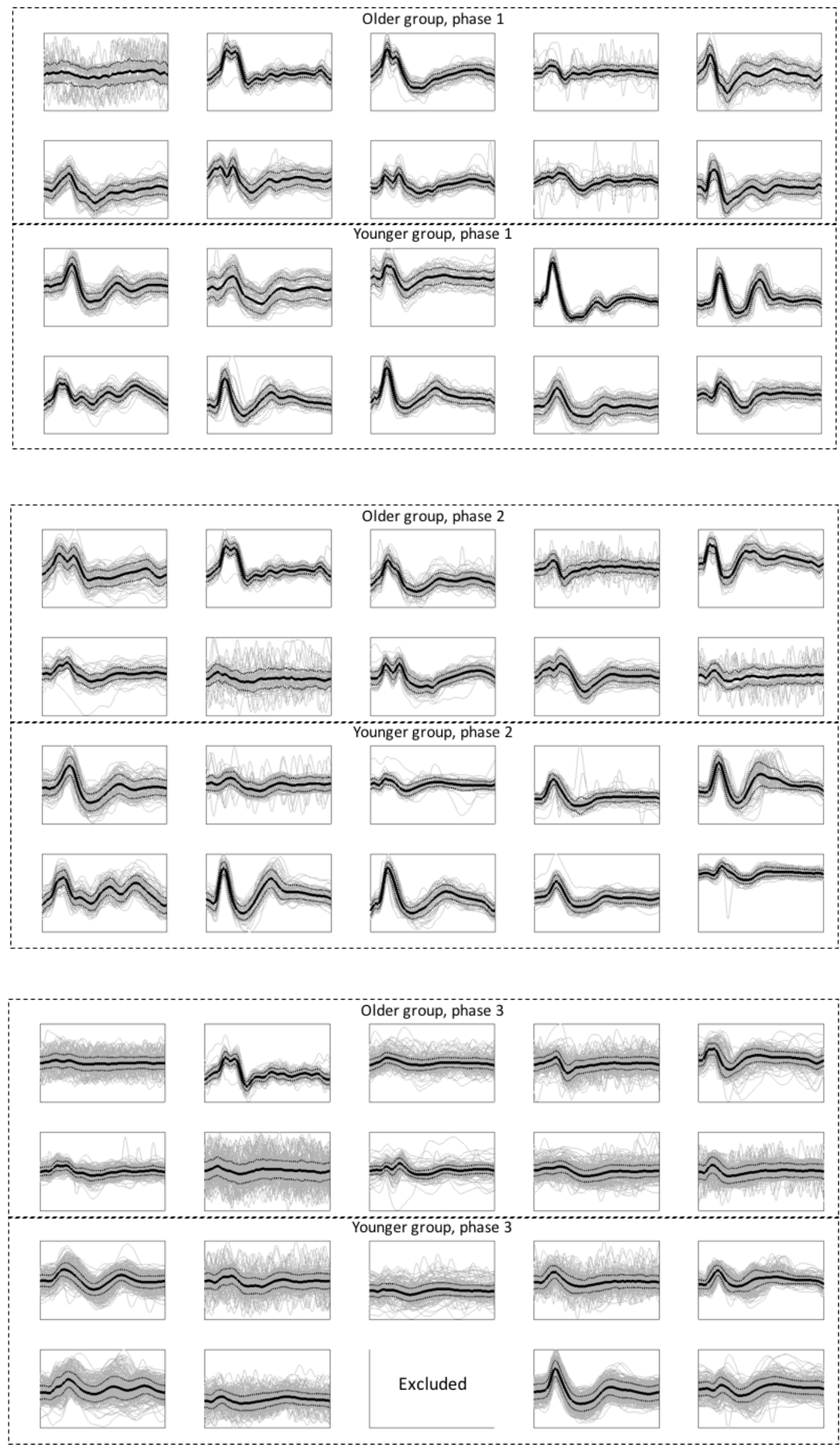

Fig. 2. ICG (-dZ/dt) waveforms for each beat, resampled to an even length of one second (gray lines). The ensemble averages for every individual and phase is shown in the thick black line with \pm 1 standard deviation in the dashed lines. 

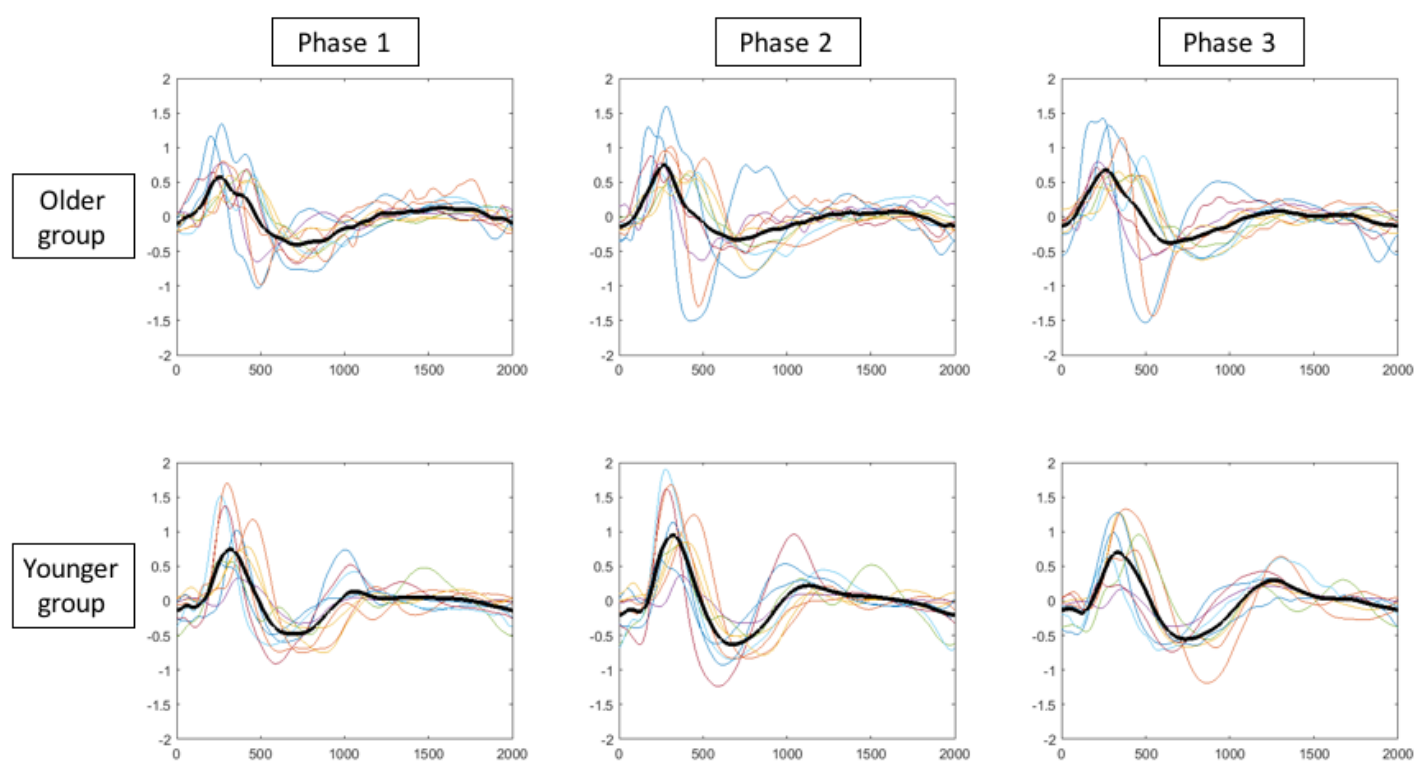

Fig. 3. The ensemble averages of $-d z / d t$ for each individual within each phase (colored lines). The mean of these curves is shown in the thick black lines (grand ensemble averages).
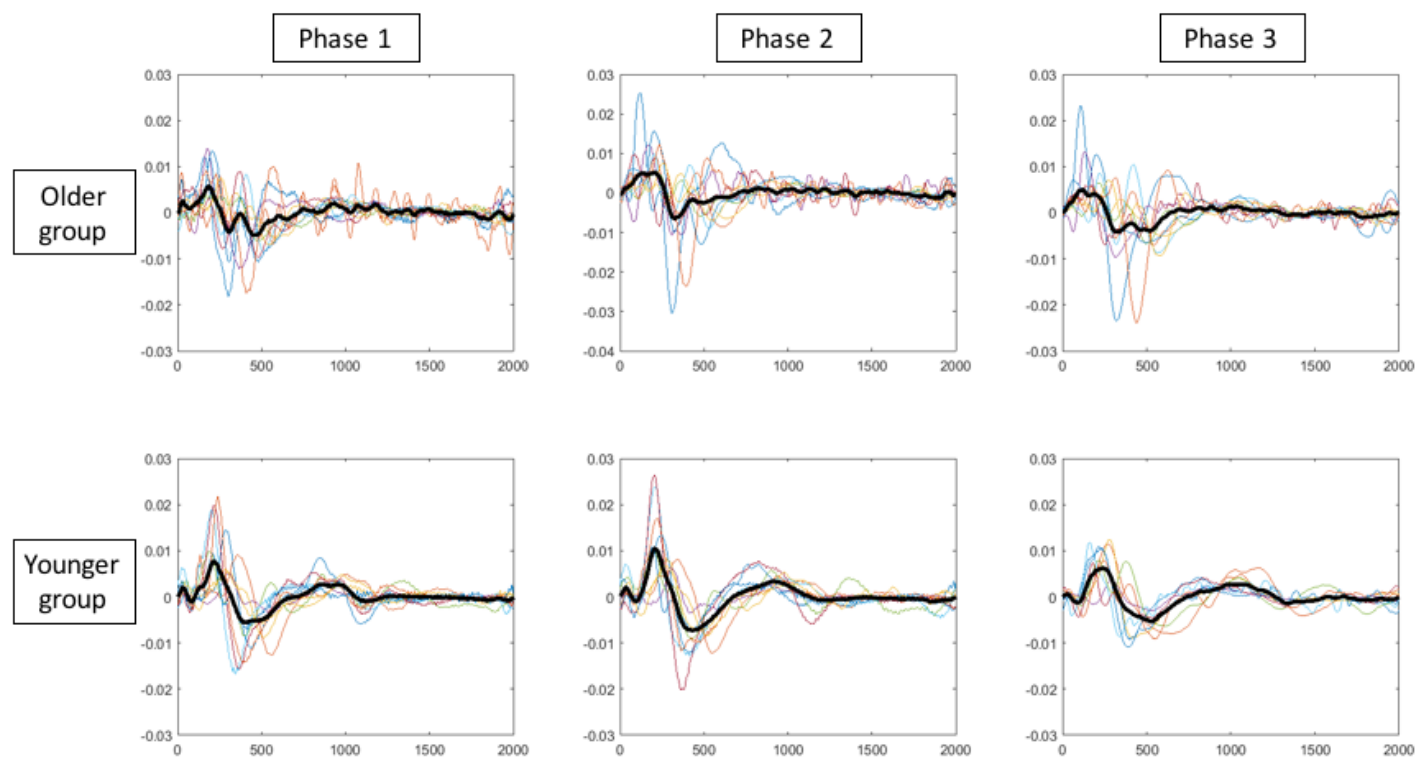

Fig. 4: The ensemble averages of $-d^{2} z / d t^{2}$ for each individual within each phase (colored lines). The mean of these curves is shown in the thick black lines (grand ensemble averages).

However, other researchers have reported no agerelated differences in the pre-ejection period in a study on 133 healthy participants between 30 and 70 years old [Uchino et al., 1999].

Ermishkin et al. [2012] found that healthy subjects at the age below 40 had typical ICG waveforms (as depicted in frequently cited publications), while they found abnormal ICG waveforms in older subjects (above 60). This alteration is seen as a stepwise pre-ejection wave superposing the ejection wave front in the impedance signal, giving rise to double-humped $\mathrm{dz} / \mathrm{dt}$ and $\mathrm{d}^{2} \mathrm{Z} / \mathrm{dt}^{2}$ curves when the preejection changes become especially pronounced. This is in agreement with our recordings, where many of the older subjects show a double-humped $\mathrm{dZ} / \mathrm{dt}$ curve during the $\mathrm{C}$ wave, while this is not clearly present in any of the younger subjects (figure 2). The examples in Ermishkin et al. [2012] also shows an earlier upward deflection of the ICG signal in the older group, in agreement with our averaged ICG waveforms (figure 3). As discussed in Ermishkin et al. [2014], it is not clear whether these abnormal ICG waveforms are of pathological of physiological nature, and that the underlying mechanisms of such phenomena remain unclear and require further research [Ermishkin et al. 2014].

Most other studies have used an electrode setup that is slightly different from the setup used for acquisition of the ICG recordings analyzed in this study, which could possibly 
cause a small difference in the ICG waveform. The differences between the setups is clearly shown in Mansouri [2018], figure 1 where the setup in $b$ was used for the recordings in this study.

This analysis has several limitations and a weak ground for drawing general conclusions on the age-related cardiovascular physiology. The sample is too small to know whether the presented average waveforms represent the typical waveform for the age groups. The average waveforms can thus not be used as reference material for ICG morphology in the two age groups. The recordings were done with an electrode configuration slightly different from the conventional one (see figure 1 in [Aria et al., 2019]), and the possibility for comparison with other studies could be limited due to this difference, as the electrode configuration may affect the ICG recording. Inspecting figure 1, it is clear that some recordings had weak or noisy ICG signals, seen by e.g. many lines far outside the \pm 1 standard deviation band, indicating that technical issues could have affected the quality of the data.

However, the results indicate a possible association between ICG morphology and aging. Relating this to physiology, the most common cardiovascular change with aging is arteriosclerosis. Derived parameters from impedance cardiography has been used in assessment of arterial compliance [Medina-Lazama et al., 2018], and a relation between arterial stiffness and ICG morphology could also be possible.

While there is an obvious need of hemodynamic monitoring of the critically ill, most of the existing methods have their limitations. Among the most frequently used methods, the pulmonary artery catheter is still widely used despite a decline in use in the recent years due to the lack of improved outcome and fear for infections [Vincent, 2008; Gershengorn, 2013]. Thermodilution is still regarded as the gold standard, and other noninvasive methods are compared to this [Josteen, 2017]. The problem with the noninvasive methods has been the accuracy. For bioimpendance measurement, the percentage error in COmeasuring devices was found to be $42 \%$ in a recent metanalysis [Josteen, 2017]. Ultrasound is a noninvasive method with few side effects, but the esophageal Doppler has limited accuracy in measurement of cardiac output and echocardiography has limited duration of placement and is operatordependent [Sakka, 2015]. Pulse pressure monitors and plethysmography do also have shortcomings, like for bioimpedance measurements [Sakka, 2015]. An interesting question is whether ICG measurements have the potential of providing continuous measurements of the most important hemodynamics at low cost with no invasive elements and with sufficient accuracy. There are many limitations that increase the uncertainty and reduce the reproducibility of the ICG-measurements; electrode placement, electrical interference from surrounding equipment and long-term electrode characteristics are some of them. However, this study suggests that the ICG morphology is related to aging, and possibly pathology such as stiffening of arteries.

Further research should include a larger sample of younger and older subjects to check the reproducibility of these findings, including reference measurements for arterial stiffness (such as carotid-femural pulse wave velocity) and blood pressure. As brought up in Ermishkin et al. [2012], morphological waveform variability may hamper accurate identification of characteristic points on the ICG recording, and more knowledge on natural waveform variability with age may improve ICG interpretation.

As demonstrated for the ECG signal, large amounts of labeled recordings has enabled the use of deep learning to identify patterns in the waveform related to pathology such as atrial fibrillation [Attia et al., 2019]. Machine learning approaches could also be relevant for ICG recordings given the availability of larger amounts of labeled data.

\section{Conclusion}

Although this study is small and lacks solid conclusions, the reported findings contribute to the scarce literature on agerelated changes in the ICG morphology. This study indicates that there is a measurable difference in ICG morphology between young and older persons, and that the measurable difference is most probably a result of the physiological changes in the vascular system as a result of stiffening arteries. It is unclear whether this approach could improve the utility of ICG in clinical settings, but we believe that further research on ICG morphology could possibly improve the method.

\section{Conflict of interest}

Authors state no conflict of interest.

\section{References}

Aria S, Elfarri Y, Elvegård M, Gottfridsson A, Grønaas HS, et al. Measuring Blood Pulse Wave Velocity with Bioimpedance in Different Age Groups. Sensors 2019, 19,4, 850 https://doi.org/10.3390/s19040850

Attia ZI, Noseworthy PA, Lopez-Jimenez F, Asirvatham SJ, Deshmukh AJ, et al. An artificial intelligence-enabled ECG algorithm for the identification of patients with atrial fibrillation during sinus rhythm: a retrospective analysis of outcome prediction. The Lancet. 2019, 394, 10201, P861-867 https://doi.org/10.1016/S0140-6736(19)31721-0

Benouar S, Hafid A, Attari M, Kedir-Talha M, Seoane F. Systematic variability in ICG recordings results in ICG complex subtypes steps towards the enhancement of ICG characterization, J Electr Bioimp 2018, 9, 72-82 https://doi.org/10.2478/joeb-2018-0012

Bernstein DP. A new stroke volume equation for thoracic electrical bioimpedance: theory and rationale. Crit Care Med. 1986, 14, 10, 904-909. https://doi.org/10.1097/00003246-198610000-00017

Bernstein DP. Impedance cardiography: Pulsatile blood flow and the biophysical and electrodynamic basis for the stroke volume equations. J Electr Bioimp 2009, 1,1, 2-17

https://doi.org/10.5617/jeb.51 
Bernstein DP. Impedance cardiography: Pulsatile blood flow and the biophysical and electrodynamic basis for the stroke volume equations. J Electr Bioimp 2010, 1, 1, 2-17

https://doi.org/10.5617/jeb.51

Bouza E, Alvarado N, Alcalá L, Pérez MJ, Rincón C, Muñoz P, . A randomized and prospective study of 3 procedures for the diagnosis of catheter-related bloodstream infection without catheter withdrawal. Clin Infect Dis. 2007, 44, 6, 820-826 https://doi.org/10.1086/511865

Carvalho P, Paiva RP, Henriques J, Antunes M, Quintal I, Muehlsteff J. Robust Characteristic Points for ICG - Definition and Comparative Analysis. Proceedings of the International Conference on Bio-inspired Systems and Signal Processing, Rome, Italy, 26-29 January, 2011

Chai $\mathrm{P}$, Mohiaddin R. How we perform cardiovascular magnetic resonance flow assessment using phase-contrast velocity mapping. J Cardiovasc Magn Reson. 2005, 7, 4, 705-716. https://doi.org/10.1081/jcmr-65639

Cybulski G. Impedance Cardiography. In: Ambulatory Impedance Cardiography. Lecture Notes in Electrical Engineering, vol 76. Springer, Berlin, Heidelberg, 2011

Drazner M, Thompson B, Rosenberg PB, Kaiser PA, Boehrer JD, Baldwin BJ, Dries DL, Yancy CW, Comparison of impedance cardiography with invasive hemodynamic measurements in patients with heart failure secondary to ischemic or nonischemic cardiomyopathy. Am J Cardiol, 2002, 89, 8, 993-995

Ermishkin VV, Kolesnikov VA, Lukoshkova EV, Mokh VP, Sonina RS, Dupik NV, Boitsov SA. Variable impedance cardiography waveforms: how to evaluate the preejection period more accurately. J Phys Conf Ser 407, 2012, 012016 https://doi.org/10.1088/1742-6596/407/1/012016

Ermishkin VV, Kolesnikov VA, Lukoshkova EV. Age-dependent and 'pathologic' changes in ICG waveforms resulting from superposition of pre-ejection and ejection waves. Physiol Meas, 2014, 35, 6, 943-63 https://doi.org/10.1088/0967-3334/35/6/943

Fegler G. Measurement of cardiac output in anesthetized animals by a thermo-dilution method. Q J Exp Physiol, 1954, 39, 153-164. https://doi.org/10.1113/expphysiol.1954.sp001067

Gershengorn HB, Wunsch H. Understanding changes in established practice: pulmonary artery catheter use in critically ill patients.

Crit Care Med. 2013, 41, 12, 2667-76

https://doi.org/10.1097/CCM.0b013e318298a41e

Josteen A, Desebbe O, Suehiro K, Murphy LSL, Essiet M et al.. Accuracy and precision of non-invasive cardiac outputmonitoring devices in perioperative medicine: asystematic review and metaanalysis. Br J Anaesth, 2017, 118, 3, 298-310.

https://doi.org/10.1093/bja/aew461

Kubicek WG, Karnegis JN, Patterson RP, Witsoe DA, Mattson RH. Development and evaluation of an impedance cardiac output system. Aerosp Med. 1966, 37,12, 1208-1212

Li J, Sun J, Song Y, Zhao J. Accelerating MRI reconstruction via three-dimensional dual-dictionary learning using CUDA. J Supercomput 2015, 71, 10, 2381-2396 https://doi.org/10.1007/s11227-015-1386-z
Liñares J. Diagnosis of catheter-related bloodstream infection: conservative techniques. Clin Infect Dis. 2007, 44, 6, 827-829 https://doi.org/10.1086/511885

Mansouri S, Alhadidi T, Chabchoub S, Ben Salah R. Impedance cardiography: recent applications and developments. Biomed Research, 2018, 29, 19, 3542-3552 https://doi.org/10.4066/biomedicalresearch.29-17-3479

Medina-Lezama J, Narvaez-Guerra O, Herrera-Enriquez K, MoreyVargas OL, Bolaños-SalazarJF et al. Hemodynamic Patterns Identified by Impedance Cardiography Predict Mortality in the General Population: The PREVENCION Study

J Am Heart Assoc. 2018, 7, 18

https://doi.org/10.1161/JAHA.118.009259

Pan J, Tompkins WJ. A Real-Time QRS Detection Algorithm. IEEE Trans Biomed Eng. 1985, BME-32, 230-236 https://doi.org/10.1109/TBME.1985.325532

Sakka SG. Hemodynamic monitoring in the critically ill patient current status and perspective. Front Med. 2015,2, 44, 1-6 https://doi.org/10.3389/fmed.2015.00044

Sramek BB, Rose DM, Miyamoto A. Stroke volume equation with a linear base impedance model and its accuracy, as compared to thermodilution and magnetic flowmeter techniques in humans and animals. Paper presented at: The 6th International Conference on Electrical Bioimpedance, Zadar, Yugoslavia, 1983, 38

Sun Z. Aging, Arterial Stiffness and Hypertension. Hypertension. $2015,65,2,252-256$ https://doi.org/10.1161/HYPERTENSIONAHA.114.03617

Thiele RH, Bartels K, Gan TJ. Cardiac output monitoring, a contemporary assessment and review. Crit Care Med. 2015, 43, 1, 177-185. https://doi.org/10.1097/CCM.0000000000000608

Van De Water JM, Miller TW, Vogel RL, Mount BE, Dalton ML. Impedance cardiography, the next vital sign technology. Chest. 2003, 123, 6, 2028-2033. https://doi.org/10.1378/chest.123.6.2028

Van Eijnatten MAJM, van Rijssel MJ, Peters RJA, Verdaasdonk RM, Meijer JH. Comparison of cardiac time intervals between echocardiography and impedance cardiography at various heart rates. J Electr Bioimp. 2014, 5, 2-8

Vincent JL, Pinsky MR, Sprung CL, Levy M, Marini JJ, Payen D, et al. The pulmonary artery catheter: in medio virtus. Crit Care Med. 2008, 36, 11, 3093-6. https://doi.org/10.1097/CCM.0b013e31818c10c7

Young DW. What does an MRI scan cost? Healthc Financ Manage 2015, 69, 11, 46-49 https://doi.org/10.4172/2168-9784.1000.167 